\title{
Berge's maximum theorem to vector-valued functions with some applications
}

\author{
Quu Xiaoling, Peng Dingtao*, Yu Jian
}

School of Mathematics and Statistics, Guizhou University, Guiyang 550025, China.

\author{
Communicated by S. S. Chang
}

\begin{abstract}
In this paper, we introduce pseudocontinuity for Berge's maximum theorem for vector-valued functions which is weaker than semicontinuity. We prove the Berge's maximum theorem for vector-valued functions with pseudocontinuity and obtain the set-valued mapping of the solutions is upper semicontinuous with nonempty and compact values. As applications, we derive some existence results for weakly Pareto-Nash equilibrium for multiobjective games and generalized multiobjective games both with pseudocontinuous vector-valued payoffs. Moreover, we obtain the existence of essential components of the set of weakly Pareto-Nash equilibrium for these discontinuous games in the uniform topological space of best-reply correspondences. Some examples are given to investigate our results. (C)2017 All rights reserved.
\end{abstract}

Keywords: Maximum theorem, vector-valued functions, set-valued mapping, pseudocontinuity, weakly Pareto-Nash equilibrium, essential components.

2010 MSC: 47H04, 90C31, 91A10.

\section{Introduction}

The well-known Berge's maximum theorem has significant application in many areas such as mathematical economics, game theory, optimization theory and control theory. Therefore, the topics about this theorem have been still very active and popular until now.

The well-known Berge's maximum theorem is as follows:

Theorem 1.1 ([3, Berge's Maximum Theorem]). Let $\mathrm{X}$ and $\mathrm{Y}$ be two Hausdorff topological spaces, $\mathrm{f}: \mathrm{X} \times \mathrm{Y} \rightarrow \mathrm{R}$ be a continuous function, $\mathrm{G}: \mathrm{Y} \Rightarrow \mathrm{X}$ be a continuous set-valued mapping, and for any $\mathrm{y} \in \mathrm{Y}, \mathrm{G}(\mathrm{y})$ be a compact set. Then the set-valued mapping

$$
M(y)=\left\{x \in G(y): f(x, y)=\max _{u \in G(y)} f(u, y)\right\},
$$

is upper semicontinuous and compact on $\mathrm{Y}$.

Many results about this theorem have been achieved in the literatures and the literatures therein (see $[5,7,8,18,23,24,28])$. In the early years, Dutta and Mitra [5] presented a maximum theorem for convex structures with weaker continuity requirements and applied to the problem of optimal intertemporal

\footnotetext{
*Corresponding author

Email addresses: xlqiuzsy@163.com (Qiu Xiaoling), dingtaopeng@126.com (Peng Dingtao), Jyu1@gzu .edu .cn (Yu Jian)
} 
allocation. Tian and Zhou [24] generalized Berge's maximum theorem by introducing the feasible path transfer lower semicontinuity and prove the existence of equilibrium for the abstract economy. Morgan and Scalzo [18] introduced the pseudocontinuity and studied the maximum theorem for pseudocontinuous functions and obtained the existence of Nash equilibria for $n$ persons noncooperative games with pseudocontinuous payoffs. Recently, Yu [28] generalized the maximum theorem to the continuous vectorvalued functions and proved the existence of weakly Pareto-Nash equilibria for multiobjective games and generalized multiobjective games both with continuous vector-valued payoffs. Feidenberg et al. [8] generalized Berge's maximum theorem to noncompact image sets with K-inf-compact functions in metrizable topological spaces and KN-inf-compact functions in Hausdorff topological spaces. Soon after, Feidenberg and Kasyanov [7] obtained the local Berge's maximum theorem for noncompact feasible sets and showed that it is more general than the recently established Berge's maximum theorem in other literature. Terazono and Matani [23] gave two theorems and show us two variants of the Berge's maximum theorem and the inverse of Berge's maximum theorem, where the condition of compact-valuedness of feasible solution function was replaced with other assumptions.

All the works mentioned above concentrated on how to weaken the continuous conditions of the function and the feasible set. It is natural to ask how about the maximum theorem for vector-valued functions under some weaker continuity and convexity? For this we propose this paper. In this paper, based on the works of Morgan and Scalzo [18] and Yu [28], we prove the Berge's maximum theorem for vector-valued functions with pseudocontinuity and obtain the set-valued mapping of the solutions is upper semicontinuous with nonempty and compact values. Our result is different from the present references against the pseudocontinuous vector-valued functions. As applications, we derive some existence results for weakly Pareto-Nash equilibrium for multiobjective games and generalized multiobjective games both with pseudocontinuous vector-valued payoffs. On the other hand, from the view point of stability, the notions of essential components and essential solutions have been widely used in various fields recently such as optimal solutions, Nash equilibrium, fixed points, etc. (see [4, 21, 22, 25, 27]). Motivated by [11-14], we shall give some existence of essential components of the set of weakly Pareto-Nash equilibrium for such games, which have been studied in $[26,29,30]$. Some examples are given to investigate our results.

The rest of the paper is organized as follows. In Section 2, we recall some definitions and lemmas which will be used in the later. In Section 3, we prove the generalization of Berge's maximum theorem to vector-valued functions with pseudocontinuity. And we investigate our results on both multiobjective game and generalized multiobjective game as applied examples in Section 4. In Section 5 we obtain some existence of essential components of the set of weakly Pareto-Nash equilibrium for games above. Section 6 concludes the paper finally.

\section{Preliminaries}

First we recall some definitions and lemmas which we will use.

Definition 2.1 ([18]). Let $X$ be a Hausdorff topological space and $f: X \rightarrow R$ be a function.

(1) $f$ is said to be upper pseudocontinuous at $x_{0} \in X$ if for all $x \in X$ such that $f\left(x_{0}\right)<f(x)$, we have

$$
\limsup _{y \rightarrow x_{0}} f(y)<f(x)
$$

(2) $f$ is said to be upper pseudocontinuous on $X$ if it is upper pseudocontinuous at each $x$ of $X$;

(3) $f$ is said to be lower pseudocontinuous at $x_{0} \in X$ if $-f$ is upper pseudocontinuous at $x_{0}$;

(4) $f$ is said to be lower pseudocontinuous on $X$ if it is lower pseudocontinuous at each $x$ of $X$;

(5) $f$ is said to be pseudocontinuous at $x \in X$ if $f$ is both upper pseudocontinuous and lower pseudocontinuous at $x$. $f$ is said to be pseudocontinuous on $X$ if $f$ is pseudocontinuous at each $x$ of $X$. 
Remark 2.2. Each upper (resp. lower) semicontinuous function is also upper (resp. lower) pseudocontinuous. But the converse is not true (see [18]).

The following lemma can be found from Proposition 2.1 in [19].

Lemma 2.3. Let $\mathrm{f}$ be a real-valued function defined on a Hausdorff topological space $\mathrm{X}$. Then, $\mathrm{f}$ is pseudocontinuous on $\mathrm{X}$ if and only if the following holds:

$$
\mathrm{f}(\mathrm{x})<\mathrm{f}(\mathrm{z}) \Rightarrow\left\{\begin{array}{l}
\text { there exists a neighborhood } \mathrm{N}_{\mathrm{x}} \text { of } \mathrm{x} \text { and a neighborhood } \mathrm{N}_{z} \text { of } z \\
\text { such that } \mathrm{f}\left(\mathrm{x}^{\prime}\right)<\mathrm{f}\left(\mathrm{z}^{\prime}\right) \text { for any } \mathrm{x}^{\prime} \in \mathrm{N}_{\mathrm{x}} \text { and any } z^{\prime} \in \mathrm{N}_{z}
\end{array}\right\} .
$$

According to Lemma 2.3, we can easily derive the following Lemma 2.4.

Lemma 2.4. Let $\mathrm{X}$ and $\mathrm{Y}$ be two Hausdorff topological spaces. If $\mathrm{f}: \mathrm{X} \times \mathrm{Y} \rightarrow \mathrm{R}$ is pseudocontinuous, then for any $y \in Y, x \rightarrow f(x, y)$ is also pseudocontinuous on $X$.

For the positive integer $k, R^{k}$ means the $k$ dimensional Euclidean space, denote by

$$
R_{+}^{k}=\left\{\left(x_{1}, x_{2}, \cdots, x_{k}\right) \in R^{k}: x_{i} \geqslant 0, i=1,2, \cdots, k\right\},
$$

and

$$
\operatorname{int}_{+}^{k}=\left\{\left(x_{1}, x_{2}, \cdots, x_{k}\right) \in R^{k}: x_{i}>0, i=1,2, \cdots, k\right\} .
$$

Definition 2.5. Let $X$ be a nonempty convex subset of a linear space $E, f: X \rightarrow R^{k}$ be a vector-valued function.

(1) $f$ is said to be $R_{+}^{k}$-quasiconcave on $X$ if for any $x_{1}, x_{2} \in X$, any $z \in R^{k}$, and any $\lambda \in[0,1]$, the following relationship holds ([17]):

$$
\left\{\begin{array}{l}
f\left(x_{1}\right) \in z+R_{+}^{k} \\
f\left(x_{2}\right) \in z+R_{+}^{k}
\end{array}\right\} \Rightarrow f\left(\lambda x_{1}+(1-\lambda) x_{2}\right) \in z+R_{+}^{k} .
$$

(2) $f$ is said to be $R_{+}^{k}$-quasiconcave-like if for any $x_{1}, x_{2} \in X$ and any $\lambda \in[0,1]$, the following relationship holds ([15]):

$$
\text { either } f\left(\lambda x_{1}+(1-\lambda) x_{2}\right) \in f\left(x_{1}\right)+R_{+}^{k}, \quad \text { or } f\left(\lambda x_{1}+(1-\lambda) x_{2}\right) \in f\left(x_{2}\right)+R_{+}^{k} .
$$

Lemma 2.6. If $\mathrm{f}$ is $\mathrm{R}_{+}^{\mathrm{k}}$-quasiconcave-like, then $\mathrm{f}$ is $\mathrm{R}_{+}^{\mathrm{k}}$-quasiconcave.

Proof. For any $x_{1}, x_{2} \in X$ and any $\lambda \in[0,1]$, since $f(x)=\left(f_{1}(x), \cdots, f_{k}(x)\right)$ is $R_{+}^{k}$-quasiconcave-like, without loss of generality, we may suppose that

$$
f\left(\lambda x_{1}+(1-\lambda) x_{2}\right) \in f\left(x_{1}\right)+R_{+}^{k}
$$

that is

$$
f_{\mathfrak{i}}\left(\lambda x_{1}+(1-\lambda) x_{2} \geqslant f_{\mathfrak{i}}\left(x_{1}\right) \geqslant \min \left\{f_{\mathfrak{i}}\left(x_{1}\right), f_{\mathfrak{i}}\left(x_{2}\right)\right\}, \quad \forall i=1, \cdots, k .\right.
$$

Therefore $f_{i}: X \rightarrow R$ is quasiconcave for any $i=1, \cdots, k$. By [17, Corollary 6.6], $f: X \rightarrow R^{k}$ is $R_{+}^{k}-$ quasiconcave.

Remark 2.7. If $k=1$, we can see that $f: X \rightarrow R$ being $R_{+}$-quasiconcave is equivalent to $f$ being $R_{+}{ }^{-}$ quasiconcave-like. However, when $k \geqslant 2$, the following example shows that

$f$ is $R_{+}^{k}$-quasiconcave $\nRightarrow f$ is $R_{+}^{k}$-quasiconcave-like.

Example 2.8. Let $X=[0,1]$, for all $x \in X$, define $f(x)=(-x, x) \in R^{2}$, since $-x$ and $x$ are quasiconcave, by 
$\left[17\right.$, Corollary 6.6], $f: X \rightarrow R^{2}$ is $R_{+}^{2}$-quasiconcave. Let $x_{1}=0, x_{2}=1$ and $\lambda=\frac{1}{2}$, then

$$
\begin{gathered}
f\left(x_{1}\right)=(0,0), \quad f\left(x_{2}\right)=(-1,1), \quad f\left(\frac{x_{1}+x_{2}}{2}\right)=f\left(\frac{1}{2}\right)=\left(-\frac{1}{2}, \frac{1}{2}\right), \\
\left(-\frac{1}{2}, \frac{1}{2}\right) \notin(0,0)+R_{+}^{2}, \quad\left(-\frac{1}{2}, \frac{1}{2}\right) \notin(-1,1)+R_{+}^{2},
\end{gathered}
$$

$f$ is not $R_{+}^{2}$-quasiconcave-like.

Lemma 2.9. If $\mathrm{f}$ is $\mathrm{R}_{+}^{2}$-quasiconcave-like, then for any $x_{1}, x_{2} \in X$, any $z \in R^{k}$ and any $\lambda \in[0,1]$, the following relationship holds:

$$
\left\{\begin{array}{l}
\mathrm{f}\left(\mathrm{x}_{1}\right) \notin z-\operatorname{int} R_{+}^{\mathrm{k}} \\
\mathrm{f}\left(\mathrm{x}_{2}\right) \notin z-\operatorname{int} R_{+}^{\mathrm{k}}
\end{array}\right\} \Rightarrow \mathrm{f}\left(\lambda x_{1}+(1-\lambda) x_{2}\right) \notin z-\operatorname{int} R_{+}^{k} .
$$

Proof. For any $x_{1}, x_{2} \in X$, any $z=\left(z_{1}, \cdots, z_{k}\right) \in R^{k}$ and any $\lambda \in[0,1]$, since $f$ is $R_{+}^{k}$-quasiconcave-like, without loss of generality, we may suppose that

$$
f\left(\lambda x_{1}+(1-\lambda) x_{2}\right) \in f\left(x_{1}\right)+R_{+}^{k}
$$

that is

$$
f_{i}\left(\lambda x_{1}+(1-\lambda) x_{2}\right) \geqslant f_{i}\left(x_{1}\right), \quad \forall i=1, \cdots, k .
$$

Since $f\left(x_{1}\right) \notin z$-intR $R_{+}^{k}$, there exists $i_{0}$ such that $f_{\mathfrak{i}_{0}}\left(x_{1}\right) \geqslant z_{\mathfrak{i}_{0}}$, hence $f_{\mathfrak{i}_{0}}\left(\lambda x_{1}+(1-\lambda) x_{2}\right) \geqslant z_{\mathfrak{i}_{0}}$ and $f\left(\lambda x_{1}+(1-\lambda) x_{2}\right) \notin z-$ intR $_{+}^{k}$.

We shall use the following Fan-Glicksberg fixed point theorem.

Lemma 2.10 ([6,9]). Let $\mathrm{X}$ be a nonempty convex compact subset of a Hausdorff locally convex space $\mathrm{E}, \mathcal{M}: \mathrm{X} \rightrightarrows \mathrm{X}$ be an upper semicontinuous set-valued mapping with nonempty, convex and compact values, then there exists $x^{*} \in X$ such that $x^{*} \in \mathcal{M}\left(x^{*}\right)$.

Throughout the paper, let $N=\{1,2, \cdots, n\}$ be a finite set where $n$ is a positive integer, $X_{i}$ be a nonempty subset of a Hausdorff topological space $E_{i}$ for each $i \in N$. Denote by $X=\prod_{i=1}^{n} X_{i}$ and $X_{\hat{i}}=\prod_{j \in N, j \neq i} X_{j}$. We also denote $x=\left(x_{1}, x_{2}, \cdots, x_{n}\right)$ by $\left(x_{i}, x_{\hat{i}}\right)$ where $x_{i} \in X_{i}$ and

$$
x_{\hat{i}}=\left(x_{1}, \cdots, x_{i-1}, x_{i+1}, \cdots, x_{n}\right) \in X_{\hat{i}} .
$$

\section{Generalization of Berge's maximum theorem}

In this section, we shall generalize Berge's maximum theorem to vector-valued functions with pseudocontinuity.

Theorem 3.1. Let $X$ and $Y$ be two Hausdorff topological spaces, $F: X \times Y \rightarrow R^{k}$ be a vector-valued function, where $F(x, y)=\left(F_{1}(x, y), F_{2}(x, y), \cdots, F_{k}(x, y)\right)$ for any $x \in X$ and any $y \in Y$, and for each $i=1,2, \cdots, k$, $\mathrm{F}_{\mathrm{i}}: \mathrm{X} \times \mathrm{Y} \rightarrow \mathrm{R}$ is pseudocontinuous, and let $\mathrm{G}: \mathrm{Y} \rightrightarrows \mathrm{X}$ be a continuous set-valued mapping with nonempty compact values. Define

$$
M(y)=\left\{x \in G(y): F(u, y)-F(x, y) \notin \operatorname{int} R_{+}^{k}, \forall u \in G(y)\right\}, \quad \forall y \in Y,
$$

then the set-valued mapping $\mathrm{M}: \mathrm{Y} \rightrightarrows \mathrm{X}$ is upper semicontinuous with nonempty and compact values.

Proof. For any $y \in Y$, since $x \rightarrow F_{1}(x, y)$ is pseudocontinuous and $G(y)$ is compact, by [18, Proposition 2.1], there exists $x \in G(y)$ such that $F_{1}(x, y)=\max _{u \in G(y)} F_{1}(u, y)$, that is,

$$
F_{1}(u, y)-F_{1}(x, y) \notin \operatorname{int} R_{+}^{k}, \quad \forall u \in G(y) .
$$

It follows that $F(u, y)-F(x, y) \notin$ int $R_{+}^{k}$, for any $u \in G(y)$. Hence, $M(y) \neq \emptyset$. 
Next, we prove that $M(y)$ is a compact set. Since $M(y) \subset G(y)$ and $G(y)$ is compact, we only need to prove that $M(y)$ is closed. Let $x_{\alpha} \in M(y), \alpha \in D$, with $x_{\alpha} \rightarrow x$, where $D$ is an index set, we need to prove $x \in M(y)$. Since $x_{\alpha} \in M(y), x_{\alpha} \in G(y)$. By the closeness of $G(y)$, we have $x \in G(y)$. Assume, by contradiction, that $x \notin M(y)$, then there exists $u^{\prime} \in G(y) \subset X$, such that

$$
F\left(u^{\prime}, y\right)-F(x, y) \in \operatorname{int} R_{+}^{k} \text {. }
$$

Then for each $i=1, \cdots, k$, we have

$$
F_{i}(x, y)<F_{i}\left(u^{\prime}, y\right) .
$$

Since $F_{i}: X \times Y \rightarrow R$ is pseudocontinuous, by Lemma 2.3 and Lemma 2.4, there exists a neighborhood $\mathrm{O}_{x}$ of $x$ such that

$$
F_{i}\left(x^{\prime}, y\right)<F_{i}\left(u^{\prime}, y\right), \quad \forall x^{\prime} \in O_{x}, \forall i=1, \cdots, k .
$$

Since $x_{\alpha} \rightarrow x$, there exists $\alpha_{0} \in D$ such that $x_{\alpha} \in O_{x}$ for any $\alpha \succ \alpha_{0}$. Thus $F_{i}\left(x_{\alpha}, y\right)<F_{i}\left(u^{\prime}, y\right)$ and $F\left(u^{\prime}, y\right)-F\left(x_{\alpha}, y\right) \in$ int $R_{+}^{k}$, which contradicts that $x_{\alpha} \in M(y)$. Therefore $x \in M(y)$ and $M(y)$ is a closed set.

Finally, we prove the set-valued mapping $M: Y \rightrightarrows X$ to be upper semicontinuous. We argue by contradiction. Assume that $M: Y \rightrightarrows X$ is not upper semicontinuous at $y \in Y$, then there exists an open set $\mathrm{O} \subset \mathrm{X}$ with $\mathrm{M}(\mathrm{y}) \subset \mathrm{O},\left\{\mathrm{y}_{\alpha}\right\}_{\alpha \in \mathrm{D}} \subset \mathrm{Y}$ with $\mathrm{y}_{\alpha} \rightarrow \mathrm{y}$, and $x_{\alpha} \in M\left(y_{\alpha}\right)$ for any $\alpha \in \mathrm{D}$, but $x_{\alpha} \notin \mathrm{O}$. Since $x_{\alpha} \in M\left(y_{\alpha}\right), x_{\alpha} \in G\left(y_{\alpha}\right)$. Since $G: Y \rightrightarrows X$ is upper continuous and $G(y)$ is a compact set, by [1, Theorem 17.16], $\left\{x_{\alpha}\right\}$ has an accumulated point $x \in G(y)$. Without loss of generality, we may suppose $x_{\alpha} \rightarrow x$. If $x \in M(y)$, then $x \in O$, which contradicts that $x_{\alpha} \rightarrow x, x_{\alpha} \notin O$ and $O$ is an open set. So there must be $x \notin M(y)$, consequently there exists $u_{0} \in G(y) \subset X$ such that

$$
F\left(u_{0}, y\right)-F(x, y) \in \operatorname{int} R_{+}^{k}
$$

that is,

$$
F_{i}(x, y)<F_{i}\left(u_{0}, y\right), \quad \forall i=1, \cdots, k .
$$

For $i=1,2, \cdots, k$, since $F_{i}: X \times Y \rightarrow R$ is pseudocontinuous, by Lemmas 2.3 and 2.4, there exist neighborhood $\mathrm{O}_{x}$ of $x$, neighborhood $\mathrm{U}_{y}$ of $y$ and neighborhood $\mathrm{O}_{u_{0}}$ of $\mathrm{u}_{0}$ such that

$$
\mathrm{F}_{\mathfrak{i}}\left(\mathrm{x}^{\prime}, \mathrm{y}^{\prime}\right)<\mathrm{F}_{\mathfrak{i}}\left(\mathrm{u}^{\prime}, \mathrm{y}^{\prime}\right), \quad \forall \mathrm{x}^{\prime} \in \mathrm{O}_{\mathrm{x}}, \quad \forall \mathrm{y}^{\prime} \in \mathrm{U}_{\mathrm{y}}, \quad \forall \mathrm{u}^{\prime} \in \mathrm{O}_{\mathrm{u}_{0}} .
$$

Since $y_{\alpha} \rightarrow y, O_{u_{0}} \cap G(y) \neq \emptyset$ and $G: Y \rightrightarrows X$ is lower semicontinuous at $y$, there exists $\alpha_{1} \in D$ such that $\mathrm{O}_{u_{0}} \cap \mathrm{G}\left(\mathrm{y}_{\alpha}\right) \neq \emptyset$ and $\mathrm{y}_{\alpha} \in \mathrm{U}_{\mathrm{y}}$ for any $\alpha \succ \alpha_{1}$. It follows from $x_{\alpha} \rightarrow x$ that there exists $\alpha_{2} \in \mathrm{D}$ with $\alpha_{2} \succ \alpha_{1}$ such that $x_{\alpha_{2}} \in \mathrm{O}_{x}$. Take $\mathfrak{u}_{\alpha_{2}} \in \mathrm{O}_{\mathfrak{u}_{0}} \cap \mathrm{G}\left(\mathrm{y}_{\alpha_{2}}\right)$, then we have

$$
F_{i}\left(x_{\alpha_{2}}, y_{\alpha_{2}}\right)<F_{i}\left(u_{\alpha_{2}}, y_{\alpha_{2}}\right), \quad \forall i=1, \cdots, k,
$$

and

$$
F\left(u_{\alpha_{2}}, y_{\alpha_{2}}\right)-F\left(x_{\alpha_{2}}, y_{\alpha_{2}}\right) \in \operatorname{int} R_{+}^{k} .
$$

However $x_{\alpha_{2}} \in M\left(y_{\alpha_{2}}\right)$ implies that

$$
F\left(u, y_{\alpha_{2}}\right)-F\left(x_{\alpha_{2}}, y_{\alpha_{2}}\right) \notin \operatorname{int} R_{+}^{k}, \quad \forall u \in G\left(y_{\alpha}\right),
$$

which is a contradiction. The proof is thus completed.

Remark 3.2. When $\mathrm{k}=1$ and for all $\mathrm{y} \in \mathrm{Y}, \mathrm{G}(\mathrm{y})=\mathrm{X}$, Theorem 3.1 coincides with [18, Theorem 3.1] which generalized Berge's maximum theorem to pseudocontinuous functions. Theorem 3.1 also includes [28, Lemma 4.7.3] as a special case where the vector-valued function $F: X \times Y \rightarrow R^{k}$ is continuous. 


\section{Existence of equilibria}

In this section, we will give some examples to demonstrate the applications of our results above.

\subsection{Existence of equilibria for multiobjective games}

Let $N=\{1,2, \cdots, n\}$ be the set of players. For each $i \in N$, let $X_{i}$ be the strategy set for player $i, X=$ $\prod_{i=1}^{n} X_{i}, F^{i}=\left(f_{1}^{i}, \cdots, f_{k}^{i}\right): X \rightarrow R^{k}$ be the vector-valued payoff of player $i$, where $k$ is a positive integer. This multiobjective game is denoted by $\Gamma=\left\{X_{i}, F^{i}\right\}_{i \in N}$. A strategy profile $x^{*}=\left(x_{1}^{*}, x_{2}^{*}, \cdots, x_{n}^{*}\right) \in X$ is called a weakly Pareto-Nash equilibrium of a multiobjective game $\Gamma$ if for each $i \in N$,

$$
F^{i}\left(y_{i}, x_{\hat{i}}^{*}\right)-F^{i}\left(x_{i}^{*}, x_{\hat{i}}^{*}\right) \notin \operatorname{int} R_{+}^{k}, \quad \forall y_{i} \in X_{i} .
$$

Theorem 4.1. Let the multiobjective game $\Gamma=\left\{X_{i}, F^{i}\right\}_{i \in N}$ satisfy the following conditions:

(1) for all $i \in N, X_{i}$ is a nonempty, convex and compact subset of a Hausdorff locally convex space $E_{i}$;

(2) for all $i \in N$, and for all $j=1,2, \cdots, k, f_{j}^{i}: X \rightarrow R$ is pseudocontinuous;

(3) for all $i \in N$, and for all $x_{\hat{i}} \in X_{\hat{i}}, y_{i} \rightarrow F^{i}\left(y_{i}, x_{\hat{i}}\right)$ is $R_{+}^{k}$-quasiconcave-like on $X_{i}$.

Then there exists a weakly Pareto-Nash equilibrium of $\Gamma$.

Proof. Define a set-valued mapping $\mathcal{M}: X \rightrightarrows X$ as follows

$$
\mathcal{M}(x)=\prod_{i=1}^{n} \mathcal{M}_{i}\left(x_{\hat{i}}\right), \quad \forall x \in X,
$$

where for each $i \in N$,

$$
\mathcal{M}_{i}\left(x_{\hat{i}}\right)=\left\{w_{i} \in X_{i}: F^{i}\left(y_{i}, x_{\hat{i}}\right)-F^{i}\left(w_{i}, x_{\hat{i}}\right) \notin \operatorname{int} R_{+}^{k}, \forall y_{i} \in X_{i}\right\} .
$$

We shall prove that $\mathcal{M}: X \rightrightarrows X$ is an upper semicontinuous set-valued mapping with nonempty, convex and compact values.

Firstly, for each $i \in N$ and each $x_{\hat{i}} \in X_{\hat{i}}$, let $G_{i}\left(x_{\hat{i}}\right)=X_{i}$, then by Theorem 3.1, $\mathcal{M}_{i}: X_{\hat{i}} \rightrightarrows X_{i}$ is upper semicontinuous with nonempty and compact values.

Secondly, we prove $\mathcal{M}_{i}\left(x_{\hat{i}}\right)$ to be a convex set for any $x_{\hat{i}} \in X_{\hat{i}}$. Let $w_{i}^{1}, w_{i}^{2} \in \mathcal{M}_{i}\left(x_{\hat{i}}\right)$ and $\lambda \in[0,1]$. From $w_{i}^{1}, w_{i}^{2} \in \mathcal{M}_{i}\left(x_{\hat{i}}\right)$, we know that $w_{i}^{1}, w_{i}^{2} \in X_{i}$ and that for any $y_{i} \in X_{i}$, there hold

$$
F^{i}\left(y_{i}, x_{\hat{i}}\right)-F^{i}\left(w_{i}^{1}, x_{\hat{i}}\right) \notin \operatorname{int} R_{+}^{k} \text {, and } F^{i}\left(y_{i}, x_{\hat{i}}\right)-F^{i}\left(w_{i}^{2}, x_{\hat{i}}\right) \notin \operatorname{int} R_{+}^{k} \text {, }
$$

that is,

$$
F^{i}\left(w_{i}^{1}, x_{\hat{i}}\right) \notin F^{i}\left(y_{i}, x_{\hat{i}}\right)-\operatorname{int} R_{+}^{k} \text {, and } F^{i}\left(w_{i}^{2}, x_{\hat{i}}\right) \notin F^{i}\left(y_{i}, x_{\hat{i}}\right)-\operatorname{int} R_{+}^{k} \text {. }
$$

Since $X_{i}$ is a convex set, $\lambda w_{i}^{1}+(1-\lambda) w_{i}^{2} \in X_{i}$. Since $y_{i} \rightarrow F^{i}\left(y_{i}, x_{\hat{i}}\right)$ is $R_{+}^{k}$-quasiconcave-like on $X_{i}$ for any $x_{\hat{i}} \in X_{\hat{i}}$, by Lemma 2.9, we have

$$
\mathrm{F}^{i}\left(\lambda w_{i}^{1}+(1-\lambda) w_{i}^{2}, x_{\hat{i}}\right) \notin F^{i}\left(y_{i}, x_{\hat{i}}\right)-i n t R_{+}^{k},
$$

that is,

$$
F^{i}\left(y_{i}, x_{\hat{i}}\right)-F^{i}\left(\lambda w_{i}^{1}+(1-\lambda) w_{i}^{2}, x_{\hat{i}}\right) \notin \operatorname{int} R_{+}^{k} .
$$

Hence, $\lambda w_{i}^{1}+(1-\lambda) w_{i}^{2} \in \mathcal{M}_{i}\left(x_{\hat{i}}\right)$ and $\mathcal{M}_{i}\left(x_{\hat{i}}\right)$ must be convex.

Now, we have shown that for each $i \in N, \mathcal{M}_{i}: X_{\hat{i}} \rightrightarrows X_{i}$ is upper semicontinuous with nonempty, convex and compact values, and hence $\mathcal{M}: X \rightrightarrows X$ is upper semicontinuous with nonempty, convex and compact values. It follows from Lemma 2.10 that $\mathcal{M}$ has a fixed point $x^{*} \in X$, that is, $x^{*} \in \mathcal{M}\left(x^{*}\right)$.

Finally, we shall prove that $x^{*} \in X$ is just a weakly Pareto-Nash equilibrium of the multiobjective game $\Gamma$. From $x^{*} \in \mathcal{M}\left(x^{*}\right)$, we have $x_{i}^{*} \in \mathcal{M}_{i}\left(x_{\hat{i}}^{*}\right)$ for each $i \in N$. According to the definition of $\mathcal{M}_{i}\left(x_{\hat{i}}\right)$, we have

$$
F^{i}\left(y_{i}, x_{\hat{i}}^{*}\right)-F^{i}\left(x_{i}^{*}, x_{\hat{i}}^{*}\right) \notin \operatorname{int} R_{+}^{k}, \quad \forall y_{i} \in X_{i} .
$$

Hence, $x^{*}$ is a weakly Pareto-Nash equilibrium point of the multiobjective game $\Gamma$. 
Corollary 4.2 ([28, Theorem 4.7.6]). Let the multiobjective game $\Gamma=\left\{X_{i}, F^{i}\right\}_{i \in N}$ satisfy the following conditions:

(1) for all $i \in N, X_{i}$ is a nonempty, convex and compact subset of a Hausdorff locally convex space $E_{i}$;

(2) for all $\mathrm{i} \in \mathrm{N}, \mathrm{F}^{\mathrm{i}}: \mathrm{X} \rightarrow \mathrm{R}^{\mathrm{k}}$ is continuous;

(3) for all $i \in N$, and for all $x_{\hat{i}} \in X_{\hat{i}}, y_{i} \rightarrow F^{i}\left(y_{i}, x_{\hat{i}}\right)$ is $R_{+}^{k}$-quasiconcave-like on $X_{i}$.

Then there exists a weakly Pareto-Nash equilibrium of $\Gamma$.

Corollary 4.3 ([18, Theorem 3.2]). Let the noncooperative game $\Gamma=\left\{X_{i}, f_{i}\right\}_{i \in N}$ satisfy the following conditions:

(1) for all $i \in N, X_{i}$ is a nonempty, convex and compact subset of a Hausdorff locally convex space $E_{i}$;

(2) for all $i \in N, f_{i}$ is pseudocontinuous on $X$;

(3) for all $i \in N$, and for all $x_{\hat{i}} \in X_{\hat{i}}, y_{i} \rightarrow f_{i}\left(y_{i}, x_{\hat{i}}\right)$ is quasiconcave on $X_{i}$.

Then there exists a Nash equilibrium of $x^{*}$ of $\Gamma$, that is for each $i \in N, x_{i}^{*} \in X_{i}$ and

$$
f_{i}\left(x_{i}^{*}, x_{\hat{i}}^{*}\right)=\max _{y_{i} \in X_{i}} f_{i}\left(y_{i}, x_{\hat{i}}^{*}\right) .
$$

Example 4.4. Let $N=1,2, X_{1}=X_{2}=[0,2], F^{1}=F^{2}=(f(x), g(x))$, where

$$
f(x)=\left\{\begin{array}{ll}
x_{1}^{2}+x_{2}^{2}, & x_{1}, x_{2} \in[0,1), \\
\frac{3}{2}, & x_{1}=x_{2}=1, \\
x_{1}^{2}+x_{2}^{2}+1, & x_{1}, x_{2} \in(1,2],
\end{array} \quad g(x)= \begin{cases}x_{2}^{2}, & x_{2} \in[0,1), \\
\frac{3}{2}, & x_{2}=1, \\
x_{2}^{2}+1, & x_{2} \in(1,2] .\end{cases}\right.
$$

The model of multiobjective game $\Gamma=\left\{\mathrm{X}_{1}, \mathrm{X}_{2}, \mathrm{~F}^{1}, \mathrm{~F}^{2}\right\}$ is made. It is easy to check $\Gamma$ satisfies the conditions of Theorem 4.1 and there is a weakly Pareto-Nash equilibrium $x^{*}=(2,2)$.

\subsection{Existence of equilibria for generalized multiobjective games}

Let $N=1,2, \cdots, n$ be the set of players. For each $i \in N$, let $X_{i}$ be the strategy set for player $i$, $X=\prod_{i=1}^{n} X_{i}, F^{i}=\left(f_{1}^{i}, \cdots, f_{k}^{i}\right): X \rightarrow \mathbb{R}^{k}$ be the vector-valued payoff of player $i, G_{i}: X_{\hat{i}} \rightrightarrows X_{i}$ be the feasible strategy correspondence of player $i$. This generalized multiobjective game is denoted by $\mathcal{G}=\left\{X_{i}, G_{i}, F^{i}\right\}_{i \in N}$. A strategy profile $x^{*}=\left(x_{1}^{*}, x_{2}^{*}, \cdots, x_{n}^{*}\right) \in X$ is called a weakly pareto-Nash equilibrium of the generalized multiobjective game $\mathcal{G}$ if for each $i \in N$,

$$
x_{i}^{*} \in G_{i}\left(x_{\hat{i}}^{*}\right) \text { and } F^{i}\left(y_{i}, x_{\hat{i}}^{*}\right)-F^{i}\left(x_{i}^{*}, x_{\hat{i}}^{*}\right) \notin \operatorname{int} R_{+}^{k}, \quad \forall y_{i} \in G_{i}\left(x_{\hat{i}}^{*}\right) .
$$

Theorem 4.5. Let the generalized multiobjective game $\mathcal{G}=\left\{\mathrm{X}_{i}, \mathrm{G}_{i}, \mathrm{~F}^{i}\right\}_{i \in \mathrm{N}}$ satisfy the following conditions:

(1) for all $i \in N, X_{i}$ is a nonempty, convex and compact subset of a Hausdorff locally convex space $E_{i}$;

(2) for all $i \in N$, and for all $j=1,2, \cdots, k, f_{j}^{i}: X \rightarrow R$ is pseudocontinuous;

(3) for all $\mathrm{i} \in \mathrm{N}$, and for all $\mathrm{x}_{\hat{\mathrm{i}}} \in \mathrm{X}_{\hat{\mathrm{i}}}, \mathrm{y}_{\mathrm{i}} \rightarrow \mathrm{F}^{\mathrm{i}}\left(\mathrm{y}_{\mathrm{i}}, \mathrm{x}_{\hat{\mathrm{i}}}\right)$ is $\mathrm{R}_{+}^{\mathrm{k}}$-quasiconcave-like on $\mathrm{X}_{i}$;

(4) for all $i \in N, G_{i}: X_{\hat{i}} \rightrightarrows X_{i}$ is a continuous set-valued mapping with nonempty, convex and compact values.

Then there exists a weakly Pareto-Nash equilibrium of $\mathcal{G}$.

Proof. The proof is similar to Theorem 4.1 when the strategy set $X_{i}$ is replaced by the feasible strategy set $G_{i}\left(x_{\hat{i}}\right)$ for any $i \in N$. So it can be omitted.

Similarly, we have the following Corollary 4.6 and Corollary 4.7. 
Corollary 4.6 ([28, Theorem 4.7.8]). Let the generalized multiobjective game $\mathcal{G}=\left\{\mathrm{X}_{i}, \mathrm{G}_{i}, \mathrm{~F}^{\mathrm{i}}\right\}_{\mathfrak{i} \in \mathrm{N}}$ satisfy the following conditions:

(1) for all $i \in N, X_{i}$ is a nonempty, convex and compact subset of a Hausdorff locally convex space $E_{i}$;

(2) for all $\mathrm{i} \in \mathrm{N}, \mathrm{F}^{\mathrm{i}}: \mathrm{X} \rightarrow \mathrm{R}$ is continuous;

(3) for all $i \in N$, and for all $x_{\hat{i}} \in X_{\hat{i}}, y_{i} \rightarrow F^{i}\left(y_{i}, x_{\hat{i}}\right)$ is $R_{+}^{k}$-quasiconcave-like on $X_{i}$;

(4) for all $i \in N, G_{i}: X_{\hat{i}} \rightrightarrows X_{i}$ is a continuous set-valued mapping with nonempty, convex and compact values.

Then there exists a weakly Pareto-Nash equilibrium of $\mathcal{G}$.

Corollary 4.7. Let the generalized game $\mathcal{G}=\left\{X_{i}, G_{i}, f_{i}\right\}_{i \in N}$ satisfy the following conditions:

(1) for all $i \in N, X_{i}$ is a nonempty, convex and compact subset of a Hausdorff locally convex space $E_{i}$;

(2) for all $i \in N, f_{i}$ is pseudocontinuous on $X$;

(3) for all $i \in N$, and for all $x_{\hat{i}} \in X_{\hat{i}}, y_{i} \rightarrow f_{i}\left(y_{i}, x_{\hat{i}}\right)$ is quasiconcave on $X_{i}$;

(4) for all $i \in N, G_{i}: X_{\hat{i}} \rightrightarrows X_{i}$ is a continuous set-valued mapping with nonempty, convex and compact values.

Then there exists an equilibrium $x^{*}$ of $\mathcal{G}$, that is for each $i \in N, x_{i}^{*} \in G_{i}\left(x_{\hat{i}}^{*}\right)$ and

$$
f_{i}\left(x_{i}^{*}, x_{\hat{i}}^{*}\right)=\max _{y_{i} \in G_{i}\left(x_{i}^{*}\right)} f_{i}\left(y_{i}, x_{i}^{*}\right) .
$$

Example 4.8. Let $N=1,2, X_{1}=X_{2}=[0,2], F^{1}=F^{2}=(f(x), g(x))$, where

$$
f(x)=\left\{\begin{array}{ll}
x_{1}^{2}, & x_{1}, x_{2} \in[0,1), \\
\frac{3}{2}, & x_{1}=x_{2}=1, \\
x_{1}^{2}+1, & x_{1}, x_{2} \in(1,2],
\end{array} \quad g(x)= \begin{cases}x_{2}^{2}, & x_{2} \in[0,1), \\
\frac{3}{2}, & x_{2}=1, \\
x_{2}^{2}+1, & x_{2} \in(1,2] .\end{cases}\right.
$$

The feasible strategy correspondence of play 1 is $G_{1}\left(x_{2}\right)=[0,1]$, for all $x_{2} \in[0,2]$. The feasible strategy correspondence of play 2 is $G_{2}\left(x_{1}\right)=[0,1]$, for all $x_{1} \in[0,2]$. For multiobjective game $\mathcal{G}=$ $\left\{X_{1}, X_{2}, G_{1}, G_{2}, F^{1}, F^{2}\right\}$, it is easy to check $\mathcal{G}$ satisfies the conditions of Theorem 4.5 and there is a weakly Pareto-Nash equilibrium $x^{*}=(1,1)$.

\section{Essential component of the equilibrium set}

In this section, we are interesting in the stability of the solutions of the discontinuous games. As we all know, many research works concentrated on the properties and characterizations of the solutions or approximate solutions, for example, see [2, 10, 16]. From [20], the space endowed with the uniform metric topology is not complete under the assumptions of pseudocontinuity. However, the essential component does not need the complete property. Therefore we consider the stability of the solution of the discontinuous games from the point of the essential component properties in the following.

Let $\mathrm{Y}$ be a nonempty compact convex subset of a normed linear space $\mathrm{E}$ and $\mathrm{F}: \mathrm{Y} \rightrightarrows \mathrm{Y}$ be a set-valued mapping. Denote

$\mathcal{D}=\{\mathrm{F}: \mathrm{Y} \rightrightarrows \mathrm{Y}: \mathrm{F}$ is an upper semicontiunous set-valued mapping with convex and compact values $\}$.

For any $F_{1}, F_{2} \in \mathcal{D}$, define

$$
\rho\left(F_{1}, F_{2}\right)=\sup _{y \in Y} h\left(F_{1}(y), F_{2}(y)\right),
$$

where $h$ is the Hausdorff metric defined on E. Clearly, $(\mathcal{D}, \rho)$ is a metric space. 
For each $F \in \mathcal{D}$, by Lemma 2.10, there exists $y^{*} \in Y$ such that $y^{*} \in F\left(y^{*}\right)$. For each $F \in \mathcal{D}$, we denote by $S(F)$ the set of fixed points of $F$. Then, $S: \mathcal{D} \rightrightarrows Y$ defines a set-valued mapping from $\mathcal{D}$ to $Y$.

For each $F \in \mathcal{D}$, the component of a point $y \in S(F)$ is the union of all the connected subsets of $S(F)$ containing $y$. Note that each component is a connected closed subset of $S(F)$, and thus, is connected compact, see [31]. It is easy to see that the component of two distinct points of $S(F)$ either coincide or disjoint, so that all components constitute a decomposition of $S(F)$ into connected pairwise disjoint compact subsets, i.e.,

$$
S(F)=\bigcup_{\alpha \in \Lambda} S_{\alpha}
$$

where $\Lambda$ is an index set, for each $\alpha \in \Lambda, S_{\alpha}$ is a nonempty connected compact subset of $S(F)$ and for any $\alpha, \beta \in \Lambda, \alpha \neq \beta, S_{\alpha} \cap S_{\beta}=\emptyset$.

Definition 5.1 ([31]). Let $F \in \mathcal{D}$ and $Q$ be a nonempty closed subset of $S(F)$. $Q$ is said to be an essential set of $S(F)$, if for each open set $O \supset Q$, there exists $\delta>0$ such that, for any $F^{\prime} \in \mathcal{D}$ with $\rho\left(F, F^{\prime}\right)<\delta$, we have $S\left(F^{\prime}\right) \cap O \neq \emptyset$. If the component $S_{\alpha}$ of $S(F)$ is an essential set, then $S_{\alpha}$ is said to be an essential component of $S(F)$.

The following lemma is [12, Theorem 3] or [31, Theorem 3.1].

Lemma 5.2. For any $\mathrm{F} \in \mathcal{D}, \mathrm{S}(\mathrm{F})$ possesses at least one essential component.

5.1. Essential component of the set of weakly Pareto-Nash equilibria for multiobjective games

Denote

$$
\mathcal{D}_{1}=\left\{\Gamma=\left(X_{i}, F^{i}\right)_{i \in N}: \Gamma \text { satisfies the conditions of Theorem 4.1 }\right\} \text {. }
$$

For each $\Gamma \in \mathcal{D}_{1}$, denote by $N(\Gamma)$ the set of all weakly Pareto-Nash equilibria of $\Gamma$.

The following theorem can be proved easily and thus the proof is omitted.

Theorem 5.3. For each $\Gamma \in \mathcal{D}_{1}$,

$$
\mathrm{N}(\Gamma)=\mathrm{S}(\mathcal{M})
$$

where $\mathcal{M}$ is the best-reply mapping of $\Gamma$.

For any $\Gamma_{1}, \Gamma_{2} \in \mathcal{D}_{1}$, define

$$
\rho_{1}\left(\Gamma_{1}, \Gamma_{2}\right)=\sup _{x \in X} h\left(\mathcal{M}_{1}(x), \mathcal{M}_{2}(x)\right),
$$

where $h$ is the Hausdorff metric defined on $E, \mathcal{M}_{1}\left(\right.$ resp. $\left.\mathcal{M}_{2}\right)$ is the best-reply correspondence of $\Gamma_{1}$ (resp. $\left.\Gamma_{2}\right)$. Clearly, $\mathcal{M}_{1}, \mathcal{M}_{2} \in \mathcal{D}$ and $\rho_{1}\left(\Gamma_{1}, \Gamma_{2}\right)=\rho\left(\mathcal{M}_{1}, \mathcal{M}_{2}\right)$.

Definition 5.4. Let $\Gamma \in \mathcal{D}_{1}$ and $Q$ be a nonempty closed subset of $N(\Gamma)$. Q is said to be an essential set of $\mathrm{N}(\Gamma)$, if for each open set $\mathrm{O} \supset \mathrm{Q}$, there exists $\sigma>0$ such that for any $\Gamma^{\prime} \in \mathcal{D}_{1}$ with $\rho_{1}\left(\Gamma, \Gamma^{\prime}\right)<\sigma$, we have $N\left(\Gamma^{\prime}\right) \cap O \neq \varnothing$. If the component $S_{\alpha}$ of $N(\Gamma)$ is an essential set, then $S_{\alpha}$ is said to be an essential component of $\mathrm{N}(\Gamma)$.

Theorem 5.5. For each $\Gamma \in \mathcal{D}_{1}$, there is at least one essential component of $\mathrm{N}(\Gamma)$ in the uniform topology of best-reply correspondences.

Proof. For each $\Gamma \in \mathcal{D}_{1}$, let $\mathcal{M}$ be the best-reply correspondence of $\Gamma$, then $\mathcal{M} \in \mathcal{D}$. By Lemma 5.2, let $S_{\alpha_{0}}$ be an essential component of $S(\mathcal{M})$, since $N(\Gamma)=S(\mathcal{M}), S_{\alpha_{0}}$ is a component of $N(\Gamma)$, we shall prove that $S_{\alpha_{0}}$ is an essential component of $N(\Gamma)$.

For each open set $O \supset S_{\alpha_{0}}$, since $S_{\alpha_{0}}$ is an essential component of $S(\mathcal{M})$, there exists $\sigma>0$ such that, for any $\mathcal{M}^{\prime} \in \mathcal{D}$ with $\rho\left(\mathcal{M}, \mathcal{M}^{\prime}\right)<\sigma$, we have $S\left(\mathcal{M}^{\prime}\right) \cap O \neq \varnothing$. For any $\Gamma^{\prime} \in \mathcal{D}_{1}$ with $\rho_{1}\left(\Gamma, \Gamma^{\prime}\right)<\sigma$, where $\mathcal{M}^{\prime}$ is the best-reply correspondence of $\Gamma^{\prime}$, since $N\left(\Gamma^{\prime}\right)=S\left(\mathcal{M}{ }^{\prime}\right)$, we must have $N\left(\Gamma^{\prime}\right) \cap O \neq \varnothing$ and $S_{\alpha_{0}}$ is an essential component of $\mathrm{N}(\Gamma)$. 
5.2. Essential component of the set of weakly Pareto-Nash equilibria for generalized multiobjective games

Denote

$$
\mathcal{D}_{2}=\left\{\mathcal{G}=\left(X_{i}, G_{i}, F^{i}\right)_{i \in N}: \mathcal{G} \text { satisfies the conditions of Theorem 4.5 }\right\} \text {. }
$$

For any $\mathcal{G} \in \mathcal{D}_{2}$, denote by $N(\mathcal{G})$ the set of all weakly Pareto-Nash equilibria of $\mathcal{G}$. Obviously, for each $\mathcal{G} \in \mathcal{D}_{2}$,

$$
N(\mathcal{G})=S(\mathcal{M})
$$

where $\mathcal{M}$ is the best-reply mapping of $\mathcal{G}$.

Let $\mathcal{G} \in \mathcal{D}_{2}$ and $S_{\alpha}$ be a component of $N(\mathcal{G})$. $\mathcal{M}$ is the best-reply correspondence of $\mathcal{G} . \mathcal{M} \in \mathcal{D}$ and

$$
N(\mathcal{G})=S(\mathcal{M}) \text {. }
$$

For any $\mathcal{G}_{1}, \mathcal{G}_{2} \in \mathcal{D}_{2}$, define

$$
\rho_{2}\left(\mathcal{G}_{1}, \mathcal{G}_{2}\right)=\sup _{x \in X} h\left(\mathcal{M}_{1}(x), \mathcal{M}_{2}(x)\right),
$$

where $h$ is the Hausdorff metric defined on $E, \mathcal{M}_{1}$ (resp. $\mathcal{M}_{2}$ ) is the best-reply correspondence of $\Gamma_{1}$ (resp. $\left.\Gamma_{2}\right)$. Clearly, $\mathcal{M}_{1}, \mathcal{M}_{2} \in \mathcal{D}$ and $\rho_{2}\left(\mathcal{G}_{1}, \mathcal{G}_{2}\right)=\rho\left(\mathcal{M}_{1}, \mathcal{M}_{2}\right)$.

Definition 5.6. Let $\mathcal{G} \in \mathcal{D}_{2}$ and $\mathrm{Q}$ be a nonempty closed subset of $N(\mathcal{G})$. $Q$ is said to be an essential set of $N(\mathcal{G})$, if for each open set $O \supset Q$, there exists $\sigma>0$ such that for any $\mathcal{G}^{\prime} \in \mathcal{D}_{2}$ with $\rho_{2}\left(\mathcal{G}, \mathcal{G}^{\prime}\right)<\sigma$, we have $N\left(\Gamma^{\prime}\right) \cap O \neq \varnothing$. If the component $S_{\alpha}$ of $N(\mathcal{G})$ is an essential set, then $S_{\alpha}$ is said to be an essential component of $\mathrm{N}(\mathcal{G})$.

Similarly the proof of Theorem 5.5, we can prove the following existence Theorem 5.7 and thus the proof is omitted.

Theorem 5.7. For each $\mathcal{G} \in \mathcal{D}_{2}$, there is at least one essential component of $\mathrm{N}(\mathcal{G})$ in the uniform topology of best-reply correspondences.

\section{Conclusions}

Berge's maximum theorem is a fundamental and important result in optimization theory, game theory and mathematical economics. In this paper, we present its new version for vector-value functions with pseudocontinuity and demonstrate its key role in the existence of equilibria for multiobjective games and generalized multiobjective games. Obviously, our results are established for vector-valued payoff function with weaker continuity and stronger convexity, so the results cannot include many present results in the literatures. What is more, Theorem 5.5 and Theorem 5.7 on the essential components of the equilibrium sets are derived in the uniform topology of best-reply correspondences, which are different from the existence results in $[12,13,26,29,30]$ in the uniform topology of payoff functions and feasible strategy correspondences. The following two examples will show that the two kinds of topology are not equivalent.

Let $N=\{1,2\}, X_{1}=[0,1], X_{2}=[0,1], X=X_{1} \times X_{2}, f=\left(f_{1}, f_{2}\right), g=\left(g_{1}, g_{2}\right)$. The uniform topology of payoff functions is defined as

$$
\rho(f, g)=\sup _{\left(x_{1}, x_{2}\right) \in[0,1] \times[0,1]}\left[\left|f_{1}\left(x_{1}, x_{2}\right)-g_{1}\left(x_{1}, x_{2}\right)\right|+\left|f_{2}\left(x_{1}, x_{2}\right)-g_{2}\left(x_{1}, x_{2}\right)\right|\right],
$$

while the uniform topology of best-reply correspondences is defined as

$$
h(F, G)=\sup _{\left(x_{1}, x_{2}\right) \in[0,1] \times[0,1]} h\left(F\left(x_{1}, x_{2}\right), G\left(x_{1}, x_{2}\right)\right),
$$

where $h$ is the Hausdorff metric, $F\left(x_{1}, x_{2}\right)=F_{1}\left(x_{2}\right) \times F_{2}\left(x_{1}\right), G\left(x_{1}, x_{2}\right)=G_{1}\left(x_{2}\right) \times G_{2}\left(x_{1}\right)$, and

$$
F_{1}\left(x_{2}\right)=\left\{x_{1} \in[0,1]: f_{1}\left(x_{1}, x_{2}\right)=\max _{\mathfrak{u}} f_{1}\left(u, x_{2}\right)\right\}, \quad \forall x_{2} \in[0,1],
$$




$$
\begin{array}{ll}
F_{2}\left(x_{1}\right)=\left\{x_{2} \in[0,1]: f_{2}\left(x_{1}, x_{2}\right)=\max _{\mathfrak{u}} f_{2}\left(x_{1}, u\right)\right\}, & \forall x_{1} \in[0,1], \\
G_{1}\left(x_{2}\right)=\left\{x_{1} \in[0,1]: g_{1}\left(x_{1}, x_{2}\right)=\max _{\mathfrak{u}} g_{1}\left(u, x_{2}\right)\right\}, & \forall x_{2} \in[0,1], \\
G_{2}\left(x_{1}\right)=\left\{x_{2} \in[0,1]: g_{2}\left(x_{1}, x_{2}\right)=\max _{\mathfrak{u}} g_{2}\left(x_{1}, u\right)\right\}, & \forall x_{1} \in[0,1] .
\end{array}
$$

Example 6.1. For all $x_{1} \in[0,1]$, and for all $x_{2} \in[0,1]$, let $f=\left(f_{1}, f_{2}\right)=(0,0)$,

$$
f^{n}=\left(f_{1}^{n}, f_{2}^{n}\right)=\left(\frac{1}{n} x_{1}-\frac{1}{n}, \frac{1}{n} x_{1}-\frac{1}{n}\right), \quad \forall n=1,2 \cdots .
$$

Then

$$
\rho\left(f, f^{n}\right)=\sup _{\left(x_{1}, x_{2}\right) \in[0,1] \times[0,1]}\left(\left|\frac{1}{n} x_{1}-\frac{1}{n}\right|+\left|\frac{1}{n} x_{1}-\frac{1}{n}\right|\right)=\frac{2}{n} \rightarrow 0 .
$$

On the other hand, $F\left(x_{1}, x_{2}\right)=F_{1}\left(x_{2}\right) \times F_{2}\left(x_{1}\right)=[0,1] \times[0,1]$ and $F^{n}\left(x_{1}, x_{2}\right)=F_{1}^{n}\left(x_{2}\right) \times F_{2}^{n}\left(x_{1}\right)=\{1\} \times[0,1]$ for any $x_{1}, x_{2} \in[0,1]$, so that

$$
h\left(F, F^{n}\right)=\sup _{\left(x_{1}, x_{2}\right) \in[0,1] \times[0,1]} h([0,1] \times[0,1],\{1\} \times[0,1])=1 .
$$

Thus $\rho\left(f, f^{n}\right) \rightarrow 0$, but $h\left(F, F^{n}\right)=1$.

Example 6.2. For all $x_{1} \in[0,1]$, and for all $x_{2} \in[0,1]$, let $f=\left(f_{1}, f_{2}\right)=(0,0), f^{n}=\left(f_{1}^{n}, f_{2}^{n}\right)=(1,1)$ for each $n=1,2 \cdots$. Then

$$
\rho\left(f, f^{n}\right)=2 .
$$

On the other hand, $F\left(x_{1}, x_{2}\right)=F_{1}\left(x_{2}\right) \times F_{2}\left(x_{1}\right)=[0,1] \times[0,1]$ and $F^{n}\left(x_{1}, x_{2}\right)=F_{1}^{n}\left(x_{2}\right) \times F_{2}^{n}\left(x_{1}\right)=[0,1] \times[0,1]$ for any $x_{1}, x_{2} \in[0,1]$, so that

$$
h\left(F, F^{n}\right)=0 .
$$

Thus $h\left(F, F^{n}\right)=0$, but $\rho\left(f, f^{n}\right)=2$.

\section{Acknowledgment}

This research was supported by the Natural Science Foundation of China (Grant No. 11401124, 71461003, 11661019) and the Science and Technology Foundation of Guizhou Province (Grant No. QKH [2016] 7425). The authors, therefore, acknowledge these supports.

\section{References}

[1] C. D. Aliprantis, K. C. Border, Infinite dimensional analysis, A hitchhiker's guide, Third edition, Springer, Berlin, (2006). 3

[2] N. L. H. Anh, P. Q. Khanh, Calculus and applications of Studniarski's derivatives to sensitivity and implicit function theorems, Control Cybernet., 43 (2014), 33-57. 5

[3] C. Berge, Topological spaces, Including a treatment of multi-valued functions, vector spaces and convexity, Translated from the French original by E. M. Patterson, Reprint of the 1963 translation, Dover Publications, Inc., Mineola, NY, (1997). 1.1

[4] O. Carbonell-Nicolau, Essential equilibria in normal-form games, J. Econom. Theory, 145 (2010), 421-431. 1

[5] P. K. Dutta, T. Mitra, Maximum theorems for convex structures with an application to the theory of optimal intertemporal allocation, J. Math. Econom., 18 (1989), 77-86. 1

[6] K. Fan, Fixed-point and minimax theorems in locally convex topological linear spaces, Proc. Nat. Acad. Sci. U. S. A., 38 (1952), 121-126. 2.10

[7] E. A. Feinberg, P. O. Kasyanov, Continuity of minima: local results, Set-Valued Var. Anal., 23 (2015), 485-499. 1

[8] E. A. Feinberg, P. O. Kasyanov, M. Voorneveld, Berge's maximum theorem for noncompact image sets, J. Math. Anal. Appl., 413 (2014), 1040-1046. 1 
[9] I. L. Glicksberg, A further generalization of the Kakutani fixed theorem, with application to Nash equilibrium points, Proc. Amer. Math. Soc., 3 (1952), 170-174. 2.10

[10] Y. Han, N.-J. Huang, Some characterizations of the approximate solutions to generalized vector equilibrium problems, J. Ind. Manag. Optim., 12 (2016), 1135-1151. 5

[11] J. Hillas, On the definition of the strategic stability of equilibria, Econometrica, 58 (1990), 1365-1390. 1

[12] J.-H. Jiang, Essential component of the set of fixed points of the multivalued mappings and its application to the theory of games, Sci. Sinica, 12 (1963), 951-964. 5, 6

[13] E. Kohlberg, J. F. Mertens, On the strategic stability of equilibria, Econometrica, 54 (1986), 1003-1037. 6

[14] Z. Lin, Essential components of the set of weakly Pareto-Nash equilibrium points for multiobjective generalized games in two different topological spaces, J. Optim. Theory Appl., 124 (2005), 387-405. 1

[15] Z. Lin, J. Yu, The existence of solutions for the system of generalized vector quasi-equilibrium problems, Appl. Math. Lett., 18 (2005), 415-422. 2.5

[16] C.-P. Liu, X.-M. Yang, Characterizations of the approximate solution sets of nonsmooth optimization problems and its applications, Optim. Lett., 9 (2015), 755-768. 5

[17] D. T. Luc, Theory of vector optimization, Lecture Notes in Economics and Mathematical Systems, Springer-Verlag, Berlin, (1989). 2.5, 2, 2.8

[18] J. Morgan, V. Scalzo, Pseudocontinuous functions and existence of Nash equilibria, J. Math. Econom., 43 (2007), $174-183$. $1,2.1,2.2,3,3.2,4.3$

[19] V. Scalzo, Hadamard well-posedness in discontinuous non-cooperative games, J. Math. Anal. Appl., 360 (2009), $697-703$. 2

[20] V. Scalzo, On the uniform convergence in some classes of non-necessarily continuous functions, Int. J. Contemp. Math. Sci., 4 (2009), 617-624. 5

[21] Q. Q. Song, On essential sets of fixed points for functions, Numer. Funct. Anal. Optim., 36 (2015), 942-950. 1

[22] Q. Q. Song, L. S. Wang, On the stability of the solution for multiobjective generalized games with the payoffs perturbed, Nonlinear Anal., 73 (2010), 2680-2685. 1

[23] Y. Terazono, A. Matani, Continuity of optimal solution functions and their conditions on objective functions, SIAM J. Optim., 25 (2015), 2050-2060. 1

[24] G. Q. Tian, J.-X. Zhou, The maximum theorem and the existence of Nash equilibrium of (generalized) games without lower semicontinuities, J. Math. Anal. Appl., 166 (1992), 351-364. 1

[25] Z. Yang, On existence and essential stability of solutions of symmetric variational relation problems, J. Inequal. Appl., 2014 (2014), 13 pages. 1

[26] H. Yang, J. Yu, Essential components of the set of weakly Pareto-Nash equilibrium points, Appl. Math. Lett., 15 (2002), 553-560. 1,6

[27] J. Yu, Essential equilibria of n-person noncooperative games, J. Math. Econom., 31 (1999), 361-372. 1

[28] J. Yu, Game theory and nonlinear analysis (Continued), Science Press, Beijing, (2011). 1, 3.2, 4.2, 4.6

[29] J. Yu, Q. Luo, On essential components of the solution set of generalized games, J. Math. Anal. Appl., 230 (1999), $303-310$. 1,6

[30] J. Yu, S.-W. Xiang, On essential components of the set of Nash equilibrium points, Nonlinear Anal., 38 (1999), $259-264$. 1,6

[31] J. Yu, H. Yang, S.-W. Xiang, Unified approach to existence and stability of essential components, Nonlinear Anal., 63 (2005), e2415-e2425. 5, 5.1, 5 